\title{
PLD4 promotes M1 macrophages to perform antitumor effects in colon cancer cells
}

\author{
LONG GAO $^{1 *}$, YAN ZHOU ${ }^{1 *}$, SHU-XIAN ZHOU ${ }^{1}$, XIAN-JING YU ${ }^{1}$, \\ JIN-MEI XU ${ }^{1}$, LUO ZUO ${ }^{1}$, YONG-HUI LUO ${ }^{2}$ and XIAO-AN LI ${ }^{1}$ \\ ${ }^{1}$ The Gastroenterology Tumor and Microenvironment Laboratory, Department of Gastroenterology, \\ The First Affiliated Hospital of Chengdu Medical College, Chengdu Medical College, Chengdu, Sichuan 610041; \\ ${ }^{2}$ Burn and Plastic Surgery Department, The First Affiliated Hospital of Chengdu Medical College, \\ Chengdu, Sichuan 610500, P.R. China
}

Received April 15, 2016; Accepted August 17, 2016

DOI: 10.3892/or.2016.5216

\begin{abstract}
Phospholipase D4 (PLD4) is a newly identified protein expressed in microglia. However, the function of PLD4 in tumor-associated macrophages (TAMs) is unknown. In the present study, we revealed that the expression of PLD4 was located in macrophages in the colon cancer mesenchymal and lymph nodes as shown by immunohistochemical analysis. Furthermore, its expression was associated with clinical staging of colon cancer. Then, THP-1 as a cell model induced into TAMs. Western blot and RT-PCR analysis showed that PLD4 was mainly presented in M1 phenotype TAMs. The secretion of pro-inflammatory cytokines in M1 macrophages was significantly reduced after the expression of PLD4 inhibited by PLD4-siRNA. Furthermore, co-cultured with condition-medium from control or PLD4-siRNA M1 macrophages for $24 \mathrm{~h}$, cell apoptosis, cycle and proliferation of cancer cells improved compared to control. These results indicated that PLD4 could be involved in the activation process of M1 phenotype macrophages.
\end{abstract}

\section{Introduction}

Colon cancer is one of the most common tumors with high cancer-related mortality rate, and millions of new cases yearly diagnosed in the world (1). Colorectal tumor environment mainly comprised endothelial, mesenchymal and immune cells. Abundant evidence indicated that immune cells played

Correspondence to: Dr Xiao-An Li, The Gastroenterology Tumor and Microenvironment Laboratory, Department of Gastroenterology, The First Affiliated Hospital of Chengdu Medical College, Chengdu Medical College, Baoguang Road 4, Xindu, Chengdu, Sichuan 610041, P.R. China

E-mail: lixa@sari.ac.cn

${ }^{*}$ Contributed equally

Key words: PLD4, pro-inflammatory cytokines, tumor-associated macrophages, colon cancer a key role in the recognition and abolishing cancer cells. The concept of cancer immune-surveillance has been supported by research showing that immune-deficiencies facilitate easy development of a neoplasm, further studies by animal models with inhibition of specific genes coding for the immune response resulted in a higher susceptibility to develop spontaneous or chemically-induced cancers $(2,3)$. Previous studies showed that the infiltration of TAMs in colorectal cancer predicted a good prognosis (4-8). TAMs have been shown to be highly plastic cells that can display both tumor preventing (M1 macrophages) and tumor promoting functions (M2 macrophages) $(9,10)$. Further studied on the distribution of M1 and M2 macrophage phenotypes in colorectal cancers showed that patients who had high numbers of infiltrating M1 macrophages obtained a significantly better prognosis (11). M1 macrophages stimulated cell-mediated responses via secreting pro-inflammatory cytokines such as IL-1, IL-6, IL-12, IL-23 and TNF- $\alpha$. Furthermore, the M1 macrophages served as a critical cellular component involved in the inflammatory response and antitumor function (12). Conversely, the M2 macrophages exerted anti-inflammatory and pro-tumorigenic activities through the production of anti-inflammatory cytokines (IL-10 and TGF- $\beta$ ) and high levels of decoys that antagonized IL-1, such as IL1RII and IL-1 receptor antagonist $(10,13)$.

Phospholipase D4 is a member of the family of phospholipid enzymes and mainly expressed in early mouse microglia. But unlike PLD1 and PLD2, PLD4 exhibited no enzymatic activity for transforming phosphatidylcholine into choline and phosphatidic acid (14). After treated with LPS, the expression of the PLD4 in microglia was increased which could promote the function of phagocytosis (15). Recent studies have indicated mutation in PLD4 gene was associated with two autoimmune diseases, named systemic sclerosis (16) and rheumatoid arthritis (17), both had abnormal inflammatory skin lesions symptoms (18). Although PLD4 was involved in microglia phagocytosis, there is little known on its function and the mechanism, thus PLD4 needed to be explored.

In the present study, we analyzed the distribution of PLD4 in colorectal cancer of different grades, clinical and pathological. Notably, the positive rate of PLD4 was associated with clinical staging of colon cancer. We observed that the 
expression of PLD4 was located in cancer tissue mesenchyme and lymph nodes. Combined with the previous study that PLD4 existed in microglia and promoted the phagocytosis of microglia after LPS stimulation, we hypothesized that TAMs were candidates. Then, the expression of PLD4 in TAMs was examined by using THP-1 as a model of macrophages. Our results demonstrated that the expression of PLD4 was closely associated with M1-type macrophages, and inhibition of its expression in M1 macrophages by siRNA led to a significant decrease in the secretion of pro-inflammatory cytokines IL-1, IL-6 and TNF- $\alpha$. Either condition medium from PLD4 siRNA-treated M1 or control-M1 cells was co-cultured with colon cancer cells. We observed significant changes in proliferation, cell cycle and apoptosis in colon cells suggesting that this protein was involved in activation of M1 cells in vitro, and promoted an inhibition effect in colon cancer.

\section{Materials and methods}

Cell culture. The THP-1, HCT116, HT29 and SW620 cell lines were cultured in $5 \% \mathrm{CO}_{2}$ at $37^{\circ} \mathrm{C}$ in RPMI-1640 supplemented with $10 \%$ fetal bovine serum (FBS). The cell lines were obtained from the American Type Culture Collection (ATCC; Manassas, VA, USA).

THP-1 macrophage preparation and condition medium collection. THP-1 cells were seeded in 6-well plates $\left(5 \times 10^{5}\right.$ cells/well). For preparation of M1-polarized THP-1 macrophages, $50 \mathrm{ng} / \mathrm{ml}$ phorbol myristate acetate (PMA; Sigma-Aldrich, St. Louis, MO, USA) was added to THP-1 cells for $48 \mathrm{~h}$, followed by $20 \mathrm{ng} / \mathrm{ml}$ interferon- $\gamma$ (IFN- $\gamma$; Peprotech, Rocky Hill, NJ, USA) and $100 \mathrm{ng} / \mathrm{ml}$ lipopolysaccharide (LPS; Sigma-Aldrich) for the following $40 \mathrm{~h}$. For preparation of M2-polarized THP-1 macrophages, $50 \mathrm{ng} / \mathrm{ml}$ PMA was added to THP-1 cells for $48 \mathrm{~h}$, followed by $20 \mathrm{ng} / \mathrm{ml}$ interleukin-4 (IL-4; Peprotech) for the following $40 \mathrm{~h}$. The M1 cells were transfected by $20 \mathrm{nM}$ siRNA against PLD4 or control siRNA (Invitrogen, Carlsbad, CA, USA) for $24 \mathrm{~h}$ with the PrimaPort siRNA transfection reagent (Invitrogen). Cell supernatants of macrophages under different conditions were collected for further research.

Flow cytometric analysis. M1 type macrophage cell markers were analyzed in flow cytometry (BD FACSCalibur flow cytometer) by using phycoerythrin (PE)-conjugated monoclonal antibody against CD86 (eBioscience) allophycocyanin (APC)-CD16 (eBioscience). Matched isotope controls were included in all experiments. Data were analyzed with the CellQuest Pro software (Tree Star, Inc., Ashland, OR, USA) after gating the macrophages population in the FSC/SSC window.

Immunohistochemical analysis. The continuous paraffin sections $(5 \mu \mathrm{m})$ were boiled in citrate buffer $(\mathrm{pH} \mathrm{6.0)}$ for $10 \mathrm{~min}$ in a microwave oven for heat-induced antigen retrieval. The sections were incubated for $1 \mathrm{~h}$ in $0.01 \mathrm{M}$ phosphatebuffered saline (PBS) that contained $10 \%$ goat serum (PBS-TGS), then overnight at $4^{\circ} \mathrm{C}$ with primary antibodies (PLD4: LifeSpan BioScience, Inc., Seattle, WA, USA; CD68: ZSGB-BIO, Beijing, China) diluted in PBS-TGS. Continuous paraffin sections from one carcinoma lymph node were used to immunostain primary antibodies PLD4 and CD68 in order to analysis the distribution of the PLD4. After washing with PBS three times, the sections were incubated with biotinylated secondary antibodies for $2 \mathrm{~h}$ at room temperature (RT). They were incubated with the ABC reagent (1:50; ZSGB-BIO) for $30 \mathrm{~min}$ at $\mathrm{RT}$, and immunoreactions were visualized using DAB kit (ZSGB-BIO) $10 \mathrm{~min}$ at RT. Images were captured by light microscopy.

Patients and tissue samples. Primary colon carcinoma tissues and their matched adjacent normal intestinal tissues were obtained from 12 colon cancer patients who underwent gastrointestinal cancer resection without preoperative treatment at the Department of Chengdu Military General Hospital and the First Affiliated Hospital of Chengdu Medical College. Signed informed consents were obtained from all patients. The study was approved by the Medical Ethics Committee of Chengdu Medical College.

Western blot analysis. Cells were scraped and centrifuged at $400 \mathrm{x} \mathrm{g}$ for $5 \mathrm{~min}$. The cell pellets were washed twice with ice-cold PBS and resuspended in ice-cold RIPA buffer [50 mM Tris-HCl, pH 7.6, $150 \mathrm{mM} \mathrm{NaCl}, 1 \% \mathrm{NP}-40,1 \%$ sodium deoxycholate, $0.1 \%$ sodium dodecyl sulfate (SDS), $1 \mathrm{mM} \mathrm{Na} \mathrm{VO}_{4}, 1 \mathrm{mM} \mathrm{NaF}$ ] supplemented with protease inhibitors. The protein content of the lysates was measured by the BCA protein assay kit. We loaded $45 \mu \mathrm{g}$ of proteins on SDS-PAGE and the separated proteins were transferred onto PVDF membranes. Blots were blocked for $2 \mathrm{~h}$ at room temperature in TBS containing 0.1\% Tween-20 (TBS-T), 5\% non-fat dry milk. After blocking, the membranes were probed overnight at $4^{\circ} \mathrm{C}$ with the primary antibody diluted in TBS-T containing 5\% non-fat dry milk. The membranes were washed four times for $20 \mathrm{~min}$ with TBS-T and probed with horseradish peroxidase-conjugated secondary antibody (mouse anti-, rabbit anti-; ZSGB-BIO) used at a 1:10,000 dilutions in TBS-T for $1 \mathrm{~h}$. After four washes for $20 \mathrm{~min}$ in TBS-T, the membranes were developed using chemiluminescent substrate and exposed to BD ECL Hyperfilm. The following primary antibodies were used in this study: rabbit anti-PLD4 antibody (LifeSpan BioScience; dilution of 1:500), and mouse anti- $\beta$ GAPDH (ZSGB-BIO; dilution of 1:1,000).

Enzyme-linked immunosorbent assay (ELISA). To determine the macrophage activation levels among the different induced conditions in vitro, the amount of IL-1, IL-8, IL-6, tumor necrosis factor- $\alpha$ (TNF- $\alpha)$ in the culture medium was measured by human instant ELISA kit (Elabscience Biotechnology, Co., Ltd., Wuhan, China), according to the manufacturer's instructions.

RT-PCR and $q R T-P C R$ analysis. Total RNA was extracted from M1 cells grown in 6-well culture plates $\left(5 \times 10^{5}\right.$ cells/well) by TRIzol Plus RNA purification kit (Invitrogen). Isolated total RNA was then reverse transcribed with Takara RNA LA $\mathrm{PCR}^{\mathrm{TM}}$ kit (AMV; Promega, Madison, WI, USA) ver.1.1. To detect the expression of PLD4, stem-loop reverse transcription PCR (RT-PCR) was performed using a qRT-PCR detection kit (Takara Bio, Shiga, Japan) according to the manufacturer's 

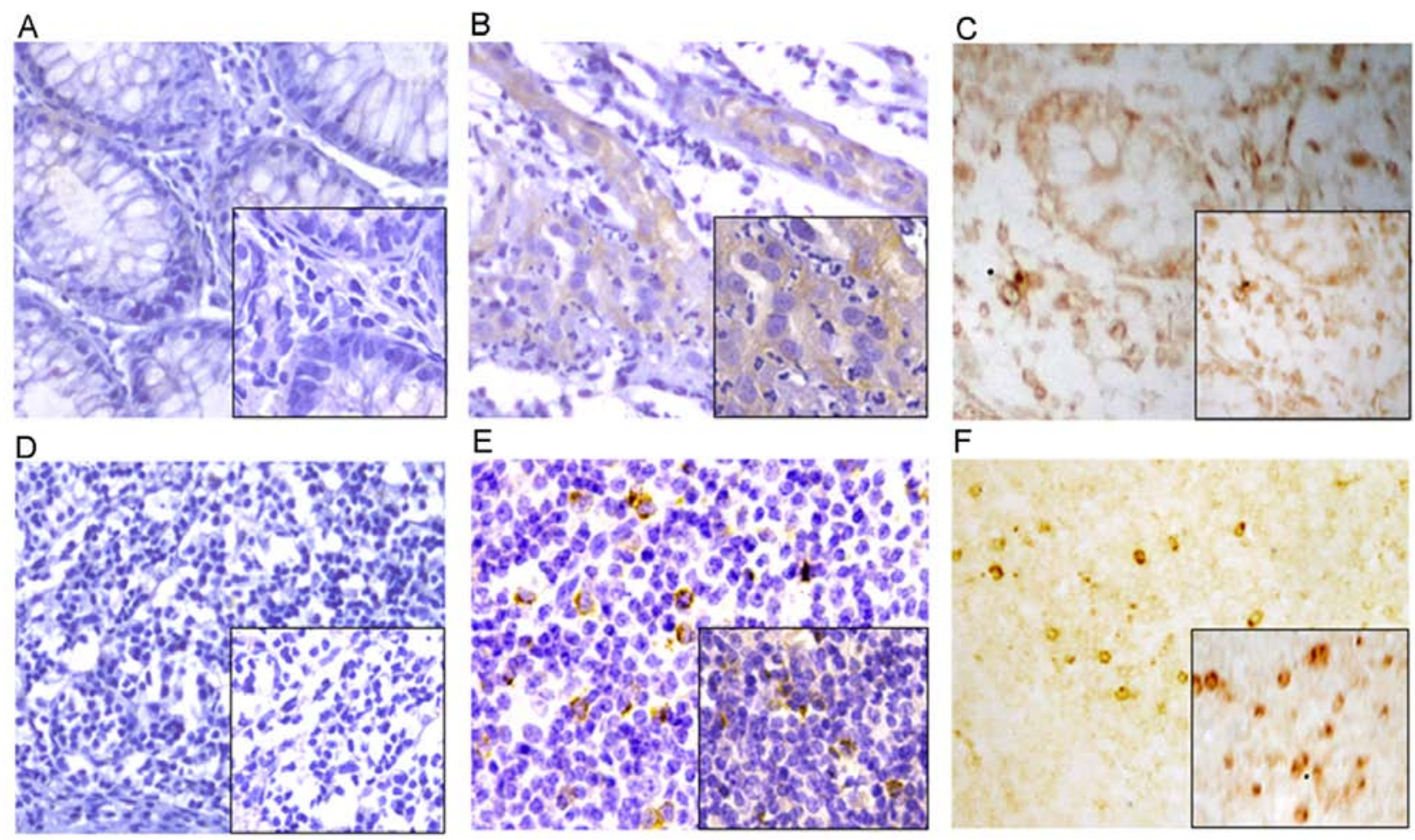

Figure 1. Sections prepared from colon cancers [B and D (with DAP): colon carcinoma, E and F (without DAP): carcinoma lymph node], and adjacent normal intestinal (A, colon, C, colon lymph node) were immunostained with antibodies against PLD4.

instructions. PLD4 and control glyceraldehyde-3-phoshate dehydrogenase (GAPDH) cDNAs (for RT-PCR) and $\beta$-actin (for qRT-PCR) were amplified by the following specific primer sets. PLD4-R, CAGGGAAATGTTCCTGCCCA and PLD4-F, CGTCCACGTGGCTTCATACT; GAPDH-R, CCCCACTTG ATTTTGGAGGGA and GAPDH-F, AGGGCTGCTTTTAA CTCTGGT; $\beta$-actin-R, GCCCACATAGGAATCCTTCTGAC and $\beta$-actin-F, AGGCACCAGGGCGTGAT.

siRNA treatment. The commercially available double-stranded siRNA oligonucleotide against PLD4 gene was purchased from Invitrogen. The M1 cells were transfected by $20 \mathrm{nM}$ siRNA against PLD4 or control siRNA (Invitrogen) for $24 \mathrm{~h}$ with the PrimaPort siRNA transfection reagent (Invitrogen), according to the manufacturer's protocol. The efficiency of siRNA to knock down the PLD4 was determined by western blot analysis and qRT-PCR.

Cell proliferation assay, cell cycle assay and apoptotic cells. HT-29, HCT116 and SW620 colon cells were plated at $10^{4}$ cells/well in a 96-well plate that contained $100 \mu 1$ RPMI-1640 supplement with $10 \%$ FBS for $24 \mathrm{~h}$ (19). Then, the culture medium was replaced by PLD4-siRNA (20 nM) group, control-group cell supernatant of M1 macrophages for another $24 \mathrm{~h}$. For cell proliferation assay, the cells were quantified using a Cell Counting kit-8 (CCK-8; Nanjing KeyGen Biotech, Co., Ltd., Nanjing, China). After 0.5, 1, 2 and $4 \mathrm{~h}$ of incubation with the reagent, each time-point absorbance at $450 \mathrm{~nm}$ was determined using a microplate reader. The measured absorbance at $0 \mathrm{~h}$ after adding supernatant was used as a standard value. For the cell cycle assay, cells were fixed at $4^{\circ} \mathrm{C}$ overnight with $70 \%$ ice-cold ethanol, washed again with PBS and incubated with $100 \mu \mathrm{l}$ RNaseA (Nanjing KeyGen Biotech) at $37^{\circ} \mathrm{C}$ for $30 \mathrm{~min}$. Staining was with $400 \mu \mathrm{l}$ propidium iodide (Nanjing KeyGen Biotech) for $15 \mathrm{~min}$ at $4^{\circ} \mathrm{C}$. The DNA content of cells was analyzed with a FACSCalibur flow cytometer (BD Biosciences) using the CellQuest software. Treated cells were harvested, washed with phosphate-buffered saline (PBS) and then centrifuged at $400 \mathrm{x} \mathrm{g}$ for $4 \mathrm{~min}$. The cell pellets were then suspended in $400 \mu \mathrm{l}$ of Annexin V-FITC/PI apoptosis detection kit (Nanjing KeyGen Biotech) at room temperature for $15 \mathrm{~min}$ in the dark. Annexin V-FITC binding was analyzed by flow cytometry $(E x=488 \mathrm{~nm}$; Em=530 nm) FL1 channel for detecting Annexin V-FITC staining and FL3 channel for detecting PI staining. Annexin V-positive, PI-negative cells were scored as early apoptotic, and double-stained cells were considered as late apoptotic.

Statistical analysis. The data were presented as the mean \pm SE. Student's t-test (two-tailed) or a one-way analysis of variance was employed to analyze the in vitro and in vivo data. Chi-square test was used to analyze the relationship between the PLD4 expression and the clinical or pathological factors.

\section{Results}

Phospholipase D4 is present in colon cancer tissues. Immunohistochemical analysis revealed that the main expression of PLD4 was located in the colon cancer mesenchymal and lymph nodes compared with normal tissues. In addition, immunohistochemistry showed strong PLD4 signals in the cytoplasm consistent with the results of previous studies (Fig. 1). Notably, PLD4 expression was associated with clinical staging of colon cancer, not pathological stage (Table I). 

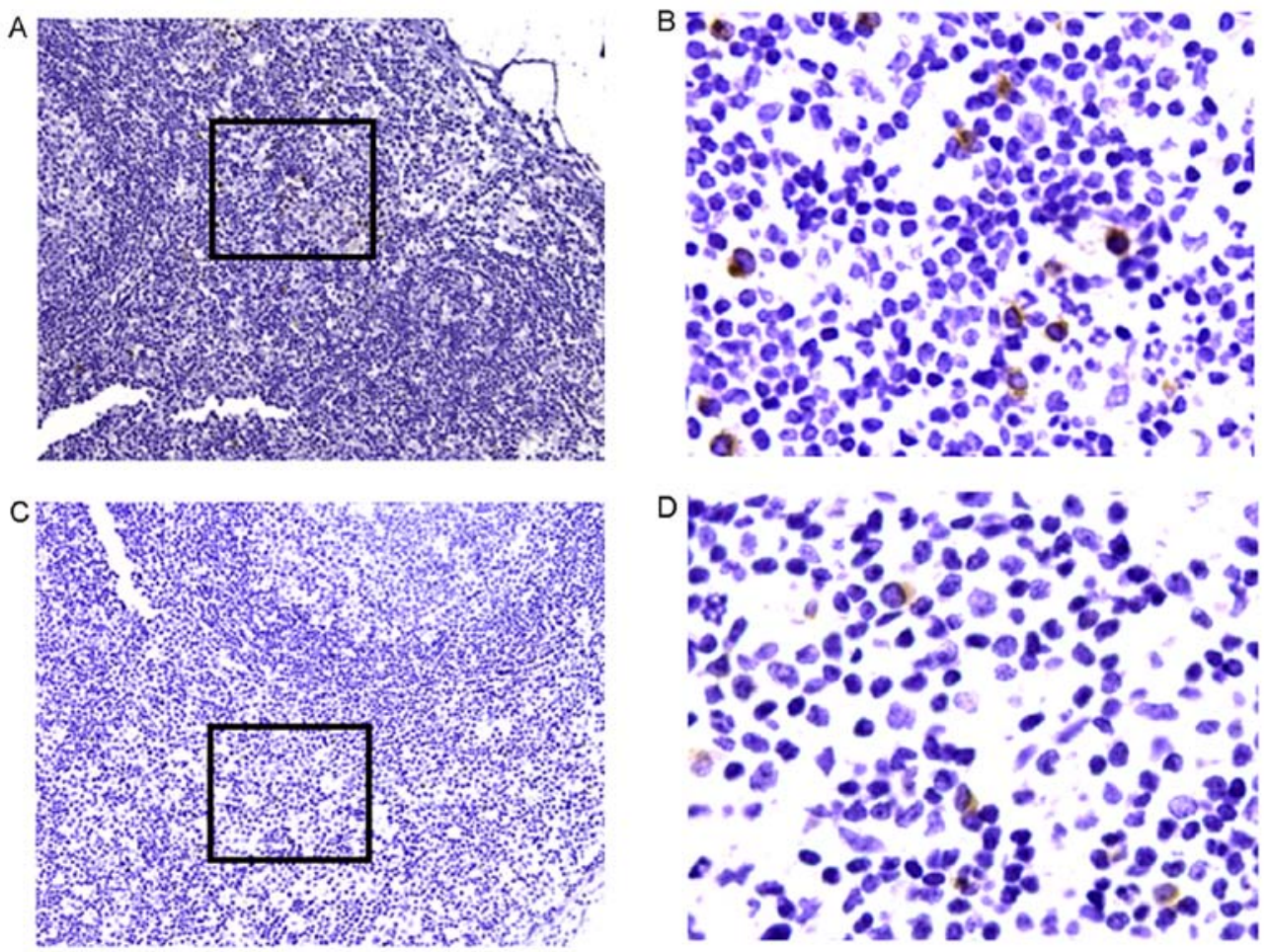

Figure 2. Continuous paraffin sections from a carcinoma lymph node were used to immunostain primary antibodies PLD4 (C and D) and CD68 (A and B) by immunohistochemical analysis in order to analyze the distribution of the PLD4. The rectangular box in the pictures represents the same position at A and C, x100 magnification; B and D, x200 magnification.

Table I. The Chi-square test results indicating that the expression of the PLD4 is related to the clinical stage $(\mathrm{P}<0.05)$.

\begin{tabular}{lrrrrr}
\hline & & \multicolumn{2}{c}{ Result } & & \\
\cline { 3 - 4 } Characteristics & $\mathrm{N}$ & Positive & Negative & P-value & $\begin{array}{c}\text { Positive } \\
\text { rate }\end{array}$ \\
\hline Age (years) & 117 & 53 & 64 & 0.969 & 0.45 \\
$<55$ & 30 & 14 & 16 & & 0.47 \\
$55-70$ & 51 & 24 & 27 & & 0.47 \\
$>70$ & 36 & 15 & 21 & & 0.42 \\
Gender & 130 & 55 & 75 & 0.996 & 0.42 \\
Male & 72 & 31 & 41 & & 0.43 \\
Female & 58 & 24 & 34 & & 0.41 \\
Histological & 100 & 37 & 63 & 0.168 & 0.37 \\
differentiation & & & & & \\
Poorly & 27 & 8 & 19 & & 0.30 \\
Moderately & 46 & 15 & 31 & & 0.33 \\
Well & 27 & 14 & 13 & & 0.51 \\
TNM stage & 109 & 56 & 53 & 0.006 & 0.51 \\
I & 40 & 29 & 11 & & 0.72 \\
II & 46 & 19 & 27 & & 0.41 \\
III & 6 & 1 & 5 & & 0.17 \\
IV & 17 & 7 & 10 & & 0.41 \\
Distant & 109 & 56 & 53 & 0.318 & 0.51 \\
metastasis & & & & & \\
Yes & 17 & 7 & 10 & & 0.41 \\
No & 92 & 10 & 43 & & 0.35 \\
\hline & & & & &
\end{tabular}

Moreover, we observed that the earlier the clinical stage, the higher positive rate of PLD4 (0.71 vs. 0.17). At the same time, we observed the relation between CD68-positive macrophages and PLD4-positive cells in continuous paraffin sections from a carcinoma lymph node, and concluded that PLD4 could exist partly in macrophages in colon cancers (Fig. 2). Then we confirmed the expression of the PLD4 in 12 human surgical specimens by western blot analysis (tumor tissues obtained without any chemotherapy). Though colon carcinoma tissues and their matched adjacent normal intestinal epithelia both exhibited the expression of PLD4 band at $48 \mathrm{kDa}$ (Fig. 3A), we found that the tissues of tumor showed higher expression of PLD4 compared with normal (Fig. 3B). The PLD4 may be related to the tumor prognosis. These results suggested that phospholipase D4 was present in differential macrophages in colon cancer.

PLD4-positive cells are the M1-polarized TAMs derived from THP-1. The above results suggested phospholipase D4 was presented in differential macrophages in colon cancers. Combined with the previous study that PLD4 existed in microglia and could enhance microglia phagocytosis after LPS stimulated, we hypothesized that M1 type TAMs were candidates in PLD4. In order to examine the expression of PLD4-positive cells, using THP-1 cells induced into tumorassociated macrophages as described (Fig. 4A). By flow cytometric analysis, the M1 macrophages showed higher levels of MHCII cell marker CD86 and CD16 compared to macrophages (20) (Fig. 4B). The expression of IL-1, IL-6, IL-8 and TNF- $\alpha$ among macrophages, M1 and M2 cells was detected by ELISA (Fig. 4C). The expression of PLD4 in 


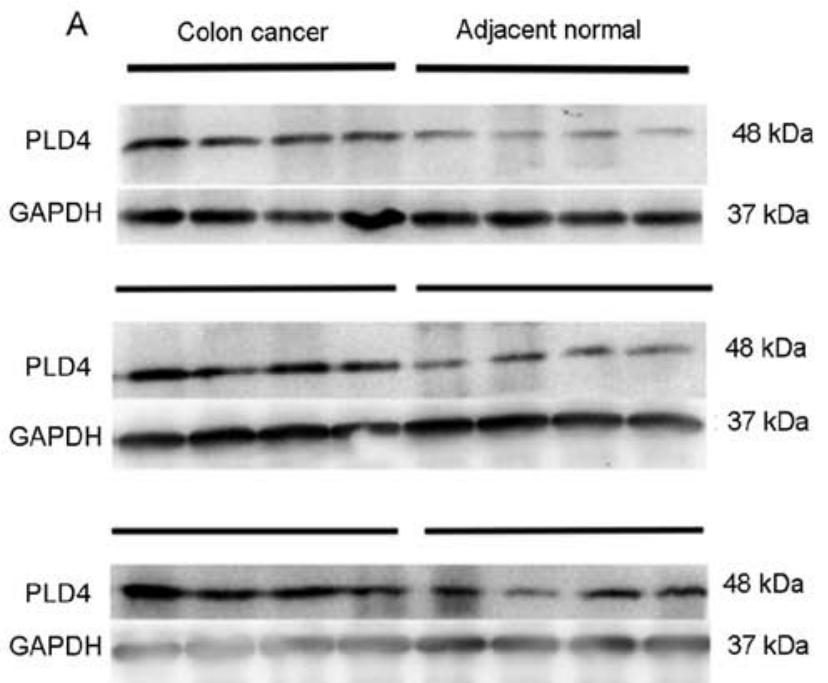

\section{B}

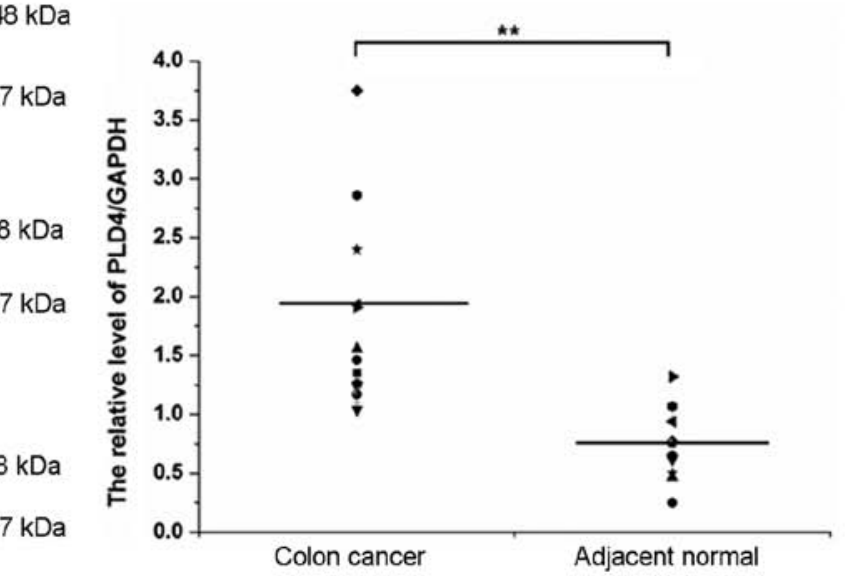

Figure 3. (A) Primary colon carcinoma tissues and their matched adjacent normal intestinal epithelia were obtained from 12 colon cancer patients who underwent gastrointestinal cancer resection without preoperative treatment. The western blot analysis (30 $\mu \mathrm{g}$ proteins, 10\% SDS-PAGE) using anti-PLD4 antibody revealed that the levels of PLD4-related bands. (B) We used the ratio of PLD4/GAPDH to show the relative level of PLD4 between colon cancers and their adjacent intestine. ${ }^{* *} \mathrm{P}<0.01$.

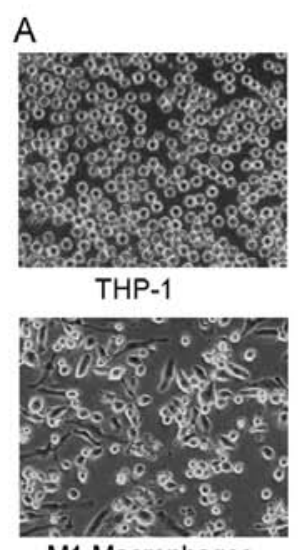

C

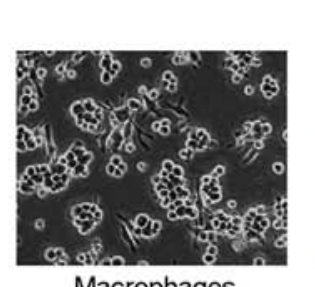

Macrophages

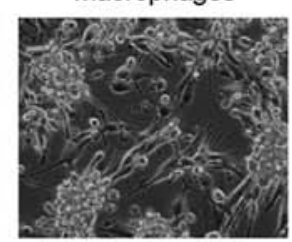

M2 Macrophages
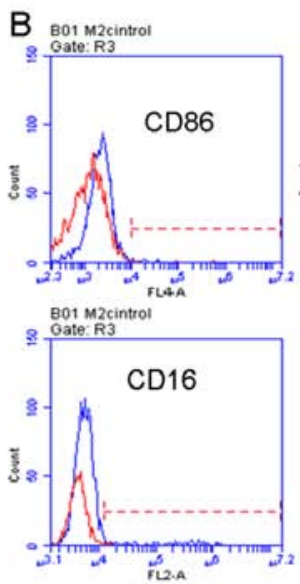

$\mathrm{MO}$
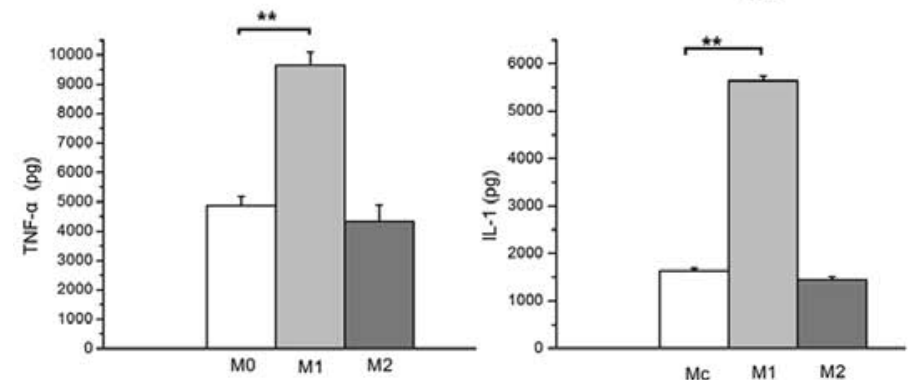
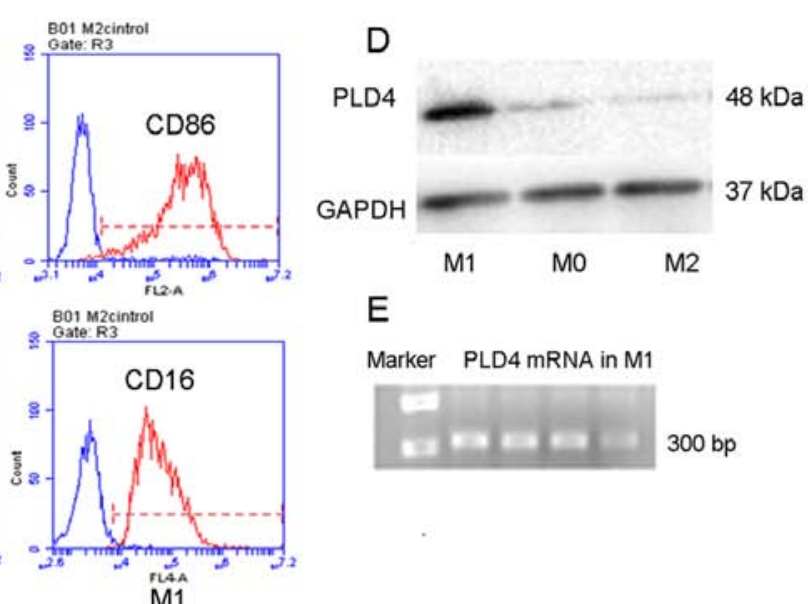

E

Marker PLD4 mRNA in M1

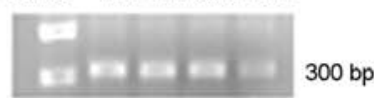

Figure 4. (A) THP-1 induces macrophages (M0), M1 macrophages and M2 macrophages. (B) The marker of PE-CD86 and APC-CD16 were used to identify the M1 macrophages by flow cytometric analysis. (C) The secretion of IL-1, IL-6, IL-8, TNF- $\alpha$ in macrophages, M1, M2 cells was detected by ELISA. (D) The western blot analysis (45 $\mu \mathrm{g}$ proteins, $10 \%$ SDS-PAGE) using anti-PLD4 antibody revealed that the levels of PLD4-related bands in LPS-stimulated M1 cells compared with those in macrophages and M2. (E) Total RNA was isolated from these cells and analyzed by RT-PCR for PLD4 mRNA expression. ${ }^{* * *} \mathrm{P}<0.01$

TAMs was confirmed by western blot analysis and RT-PCR (Fig. 4D and E). Western blot analysis of LPS plus IFN- $\gamma$ induced M1 and IL-4 induced M2 cell homogenates exhibited mainly M1 cells existed in PLD4-related bands of $48 \mathrm{kDa}$ (Fig. 4D), and the expression of PLD4 mRNA in M1 cells was confirmed by RT-PCR at bands of 300 bp (Fig. 4E). The result showed all pro-inflammation factors in M1 cells were upregulated compared the other macrophages. M1 tumor-associated macrophages expressed PLD4.

PLD4-siRNA interference reduces the activation of MI macrophages. After M1 macrophages were seeded in 6-well 
A

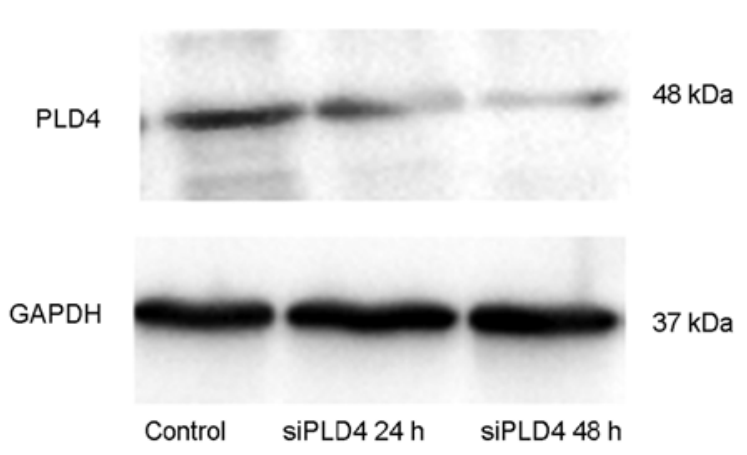

B

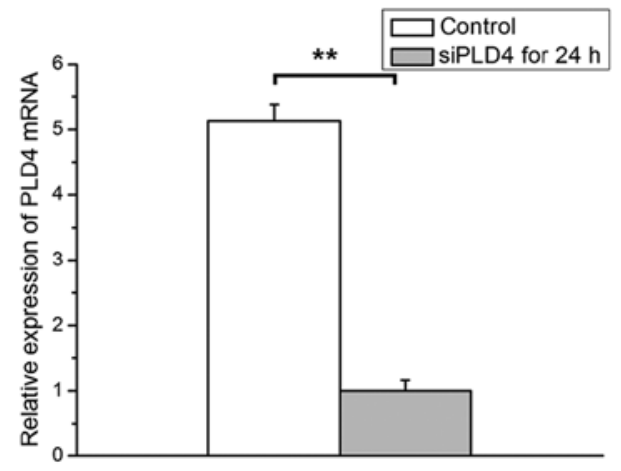

C
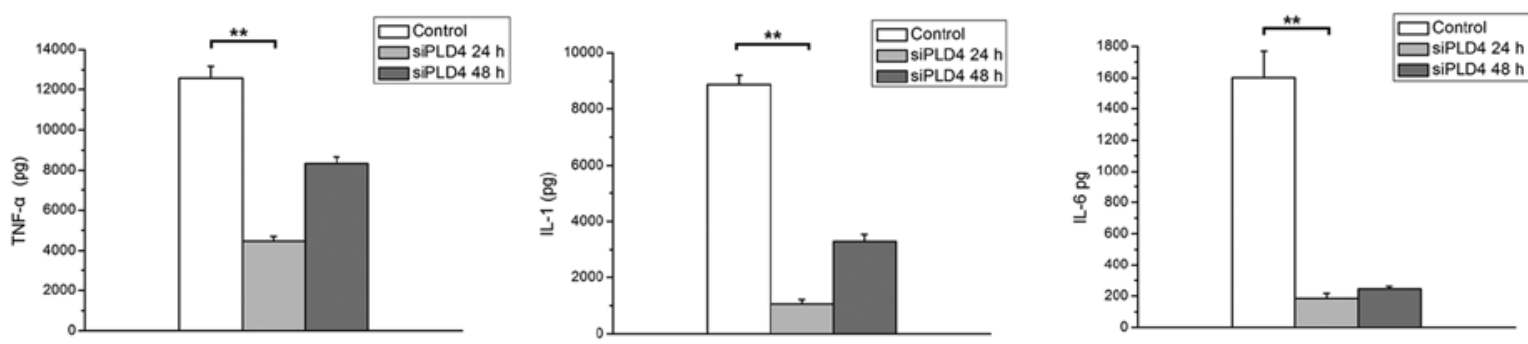

Figure 5. (A) M1 cells transfected with PLD4-siRNA or negative control (NG)-siRNA (20 nM each) for $48 \mathrm{~h}$. Total protein was collected from these cells and analyzed by western blot analysis for PLD4 and GAPDH. The relative level of PLD4 in M1 cells revealed that PLD4 was downregulated in PLD4-siRNA-treated cells compared with NG-siRNA treatment. (B) The relative PLD4 mRNA level both in PLD4-siRNA-treated cells and NG-siRNA treatment for $24 \mathrm{~h}$ was detected by qRT-PCR. M1 cells were transfected with siRNA for 24 or $48 \mathrm{~h}$. (C) After 24 and $48 \mathrm{~h}$, secretion of IL-1, IL-6 and TNF- $\alpha$ was measured by ELISA. Measurement of NG-siRNA-treated cells was used as a standard value. The data are presented as mean of three experiments. ${ }^{* *} \mathrm{P}<0.01$.

plates with siRNA $(20 \mathrm{nM})$ or vehicle for $24 \mathrm{~h}$, decreased expression of PLD4 was confirmed by western blot analysis and qRT-PCR. A marked decrease in PLD4 bands was found in siRNA-treated cells compared with control (Fig. 5A). The relative PLD4 mRNA level both in PLD4-siRNA-treated cells and NG-siRNA treatment for $24 \mathrm{~h}$ was detected by qRT-PCR (Fig. 5B). A decrease of PLD4 mRNA in PLD4-siRNAtreated cells compared with control. At the same time, the cell pro-inflammation factor levels of TNF- $\alpha$, IL-1 and IL-6 in the culture medium were detected by ELISA. The siRNA interference showed temporarily reduced release of IL-1, IL-6 and TNF- $\alpha$ in M1 cells (Fig. 5C). These results indicated that immunoreactivity of the M1 cells were inhibited.

PLD4 stimulates M1 macrophages to develop antitumor effects in colon cancer cells. We co-cultured HT-29, HCT116, SW620 colon cancer cells with the condition medium (CM) from siRNA-interfered and untreated M1 cells for $24 \mathrm{~h}$. Marked changes in tumor proliferation were observed by CCK-8 kit when HT-29, HCT116 and SW620 cells were co-cultured with two types of M1 cells and controls for $24 \mathrm{~h}$ (Fig. 6A). Using the well-described Annexin V binding assay coupled to a flow cytometric analysis allowed the distinction to be made between early (Annexin V-positive/PI-negative cells) and late (Annexin V-positive/PI-positive cells) apoptosis to determine when cell death occurred. An obvious increase in the number of apoptotic cells was noted in M1 group cells compared with control and PLD4-siRNA group
(Fig. 6B and C). At the same time, we examined the cell cycle phase changes in three types of colon cancer cells after culturing with different types of macrophages for $24 \mathrm{~h}$. The CM from M1 induced accumulation of S-phase cells in HT-29 $(\mathrm{P}<0.05)$, but in PLD4-siRNA treatment and control groups, there were no significant changes observed, compared with PLD4-siRNA and control groups, the percentage of G0/G1 cells in SW-620 were increased visibly $(\mathrm{P}<0.05)$ (Fig. 6D). However, no significant change was observed in HCT116 from each group (Fig. 6D). The three tumor cell types cultured with the inhibition of PLD4 in M1 cells showed more proliferation, shorter cell cycle and less apoptosis compared to the normal actived M1 macrophages.

\section{Discussion}

In colon cancer specimens, Forssell et al (5) suggested that TAMs prevented tumor development because patients with a high infiltration of TAMs had a better prognosis and survival rate. De-Nardo et al (21) noted that the leukocyte composition of established tumors, in which immunosuppressive M2-like TAMs likely predominated over M1 macrophages. Prada et al (22) and Wang et al (23) suggested that incipient tumors, which may be more immunogenic than established lesions, contained higher proportions of M1-like TAMs, which could initiate adaptive immune responses. In the present study, we analyzed the expression of PLD4 in 109 colon cancer specimens during different clinical and pathological grades by IHC. 

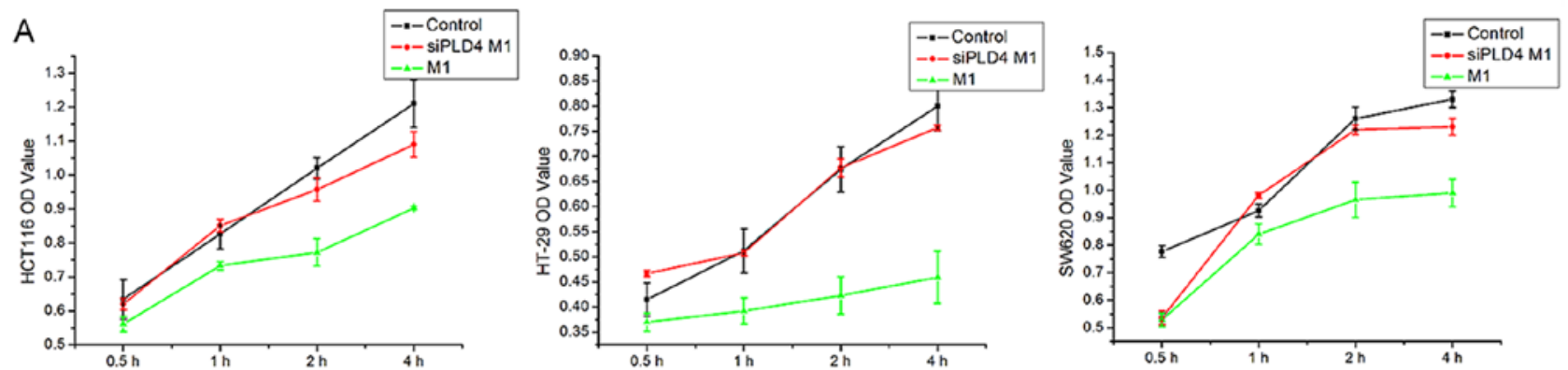

B

C
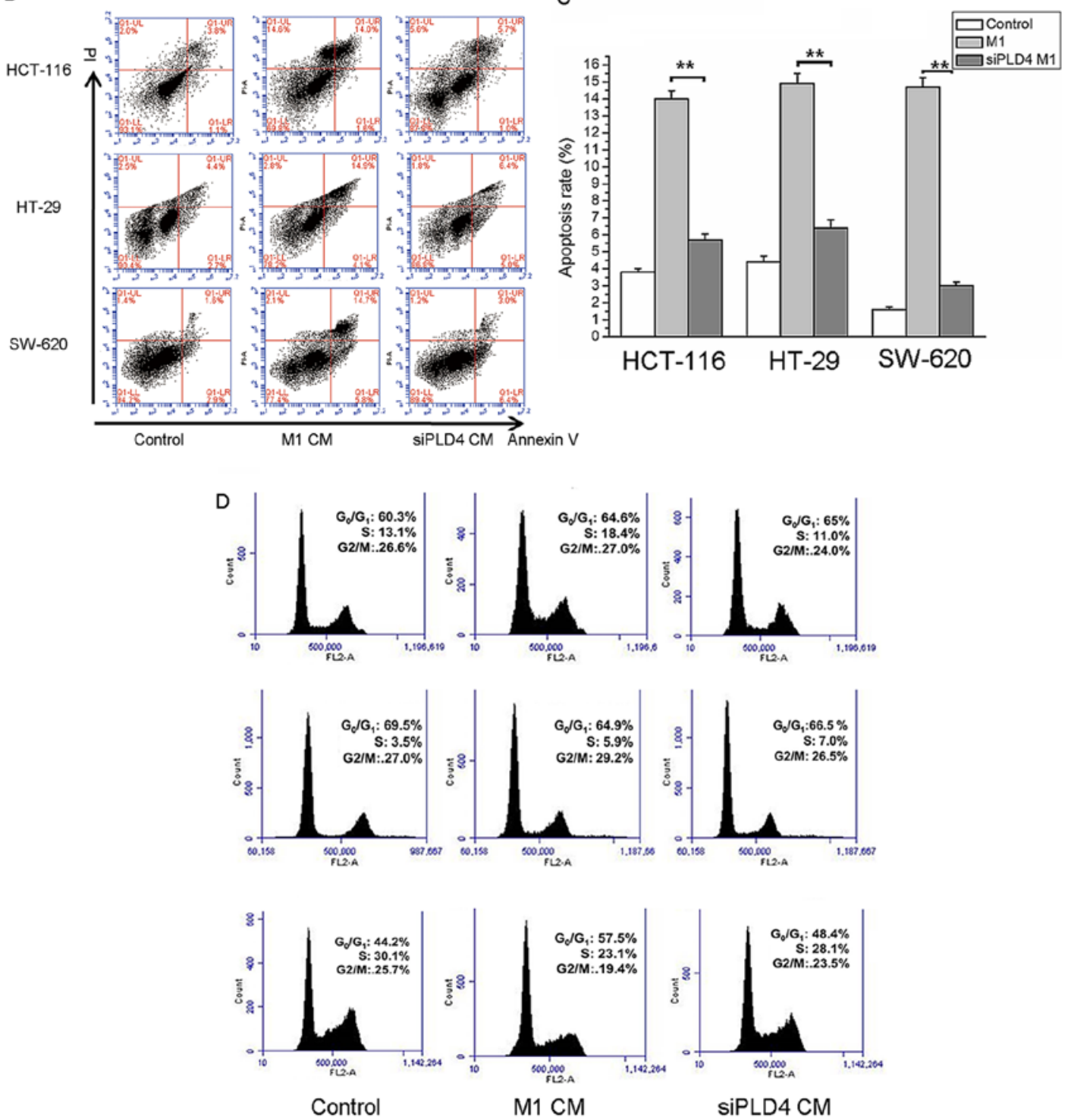

Figure 6. (A) The proliferation, (B and C) apoptotic, (D) cell cycle of HT-29, HCT116 and SW620 cells treated with condition medium (CM) from negative control, M1, siPLD4-M1 cells for $24 \mathrm{~h}$. (A) Their cell viability was determined by the CCK-8 at 0.5, 1,2 and $4 \mathrm{~h}$. The apoptotic rates (B and C) and cell cycle (D) of colon cancer cells were measured by flow cytometry. ${ }^{* *} \mathrm{P}<0.01$.

PLD4 mainly expressed in cytoplasmic region based on the immunohistochemical analysis. In addition, the results from Table I suggested that the earlier the clinical stage, the higher positive rate of PLD4. Western blot analysis in 12 human surgical tissues without any chemotherapy at early clinical stage, confirmed a surprise outcome that all of the detected samples expressed PLD4, but the tissues of tumor showed higher expression of PLD4 compared with normal. A study by Steidl et al (24) showed that tumor-associated macrophages could affect the prognosis of lymphoma in patients. Thus, we selected continuous paraffin sections from a carcinoma lymph node to analysis the relation between CD68-positive macrophages and PLD4-positive cells and showed that PLD4 existed partly in macrophages in colon cancers by immunohistochemistry (Fig. 2). The AJCC 2009 statistics showed that only in relatively early stages can surgical resection be 
conducted on patients with colon cancer (25). Moreover, we found that HCT116 and HT29 colon cancer cells did not express PLD4 (data not shown). Thus, we considered that the expression of PLD4 could be associated with the patient prognosis and existed in macrophages, but not in tumor cells. Since previous studies showed that PLD4 was present in microglia cells after treatment with LPS, it was attractively to speculate that M1 polarization TAMs in colon cancer may express PLD4, because the M1 macrophages were induced by LPS plus IFN- $\gamma$. Macrophages were a key component of the tumor-infiltrating immune cells and they could considerably affect tumor progression (21,26-28).

Considering these findings, in vitro, we induced THP-1 cells differentiated into M1 macrophages by adding phorbol myristate acetate (PMA) to THP-1 cells for $48 \mathrm{~h}$, subsequently adding IFN- $\gamma$ and LPS for the following $48 \mathrm{~h}$. In addition, M1 cells were characterized by the expression of CD86, CD16 through flow cytometric analysis. The production of pro-inflammatory factors IL-1, IL-6, IL-8 and TNF- $\alpha$ were detected by ELISA. In M1 macrophages, a higher production of IL-1, IL-6, IL-8, TNF- $\alpha$ which exerted a function of antitumor effect $(9,12)$ compared with other types of macrophages was observed. Based on these results, we suggested that the M1 macrophages were active and played an important role in antitumor progress. Subsequently, cells were harvested for western blot analysis and RT-PCR analysis. Western blot analysis ( $45 \mu \mathrm{g}$ proteins, $10 \%$ SDS-PAGE) using PLD4 antibody revealed that PLD4-positive bands were located in the $48 \mathrm{kDa}$. The expression of PLD4 mRNA was confirmed by RT-PCR. Thus, PLD4 existed in M1 macrophages originated from THP-1 cells.

Furthermore, phospholipase D4 is a member of the family of phospholipid enzymes (14), and is mainly expressed in spleen and early microglia (15). Recent studies showed two autoimmune diseases, sclerosis and rheumatoid arthritis, were related to PLD4 mutation $(16,17)$. Although PLD4 seemed to have some immunological functions, little was known about its function in cells. PLD4 might be transported extracellularly into the nucleus. We used siRNA interference to show partial inhibition of the PLD4, and showed a significant decrease in IL-1, IL-6 and TNF- $\alpha$ levels. It is known that the function of macrophages largely depends on the level of cytokine. When high levels of Th1 cytokines, such as tumor necrosis factor (TNF), IL-1 and interferon (IFNs) were present, the pro-inflammatory M1 macrophages could be established and had an antitumor effect (29). PLD4 might play an important role in regulating activation of M1 macrophages at least in stimulating macrophages increasing the release of pro-inflammatory cytokines.

To the best of our knowledge, activated M1 macrophages exerted an important role in antitumor effects. Then, we co-cultured HCT116, SW620 and HT29 colon cells with cell supernatant of M1 and si-PLD4 M1 macrophages for $24 \mathrm{~h}$. We found that colon cancer cells showed reduced proliferation, marked increased of apoptosis and cell cycle in M1 macrophages compared with si-PLD4 M1 cells. These results demonstrated that THP-1-differentiated M1 macrophages induced by LPS could release a high level of TNF- $\alpha$, IL-1, IL-6, and all were secreted into cell supernatant leading to death occurring in colon adenocarcinoma cells. Thus, PLD4 could possess a function to send signals in activation process of the M1 macrophages.
The nuclear factor- $\kappa \mathrm{B}(\mathrm{NF}-\kappa \mathrm{B})$ and the signaling transducer and activator of transcription (STAT) pathways are known to play pivotal roles in the transcriptional profile of macrophages. Among the transcriptional factors, STAT1 and canonical NF- $\mathrm{KB}$ (p50/p65 heterodimer) were essential for the M1 tumoricidal functions and triggered the expression of proinflammatory cytokines (30). LPS is a well-known activator of the NF- $\kappa \mathrm{B}$ pathway and was important in the establishment of the M1 phenotype of macrophages. The detoxified derivative of LPS had also shown promise as an adjuvant of anticancer vaccines. A number of 79 clinical trials of this drug are ongoing (31). M1-type macrophages are capable of inducing lysis in various types of cancer cells, but the mechanism of action need further exploration. It has been previously identified that more unknown proteins together with protease which could activate macrophages might be responsible for this action (32). PLD4 possibly belonged to these 'an unknown protein' and used unknown pathways to activate the macrophages. The manner of PLD4 impacted on macrophage activation still needed further study.

To conclude, the role of TAMs in tumor environment was complex since their behavior was modulated by the tumor microenvironment and various factors were involved in cross-talk that exist between TAMs and cancer cells. Indeed activated macrophages would be crucial to determine the prognosis in colon cancer. This study emphasized that PLD4 was involved in this signal network. Our findings highlighted a new pattern of tumor-associated macrophage activation in which PLD4 could play an important role in activation signal delivery. PLD4 existing in the M1 type polarization may be useful in understanding the reason high TAM density is associated with a good prognosis in colon cancer.

\section{Acknowledgements}

The present study was supported by the National Natural Science Foundation of China (grant no. 81302170), the Natural Science Foundation of Education Department of Sichuan Province (grant no. 16ZA0280), the Innovative Group Foundation of Education Department of Sichuan Province (grant no. 16TD0028), and the Natural Science Foundation of Science and Technology Department of Sichuan Province (grant no. 2016JY0090).

\section{References}

1. Jemal A, Center MM, DeSantis C and Ward EM: Global patterns of cancer incidence and mortality rates and trends. Cancer Epidemiol Biomarkers Prev 19: 1893-1907, 2010.

2. Dunn GP, Old LJ and Schreiber RD: The immunobiology of cancer immunosurveillance and immunoediting. Immunity 21 : 137-148, 2004.

3. Smyth MJ, Dunn GP and Schreiber RD: Cancer immunosurveillance and immunoediting: The roles of immunity in suppressing tumor development and shaping tumor immunogenicity. Adv Immunol 90: 1-50, 2006.

4. Algars A, Irjala H, Vaittinen S, Huhtinen H, Sundström J, Salmi M, Ristamäki R and Jalkanen S: Type and location of tumorinfiltrating macrophages and lymphatic vessels predict survival of colorectal cancer patients. Int J Cancer 131: 864-873, 2012.

5. Forssell J, Oberg A, Henriksson ML, Stenling R, Jung A and Palmqvist R: High macrophage infiltration along the tumor front correlates with improved survival in colon cancer. Clin Cancer Res 13: 1472-1479, 2007. 
6. Lackner C, Jukic Z, Tsybrovskyy O, Jatzko G, Wette V, Hoefler G, Klimpfinger M, Denk H and Zatloukal K: Prognostic relevance of tumour-associated macrophages and von Willebrand factorpositive microvessels in colorectal cancer. Virchows Arch 445: 160-167, 2004.

7. Nagorsen D, Voigt S, Berg E, Stein H, Thiel E and Loddenkemper C: Tumor-infiltrating macrophages and dendritic cells in human colorectal cancer: Relation to local regulatory $\mathrm{T}$ cells, systemic T-cell response against tumor-associated antigens and survival. J Transl Med 5: 62, 2007.

8. Zhou Q, Peng RQ, Wu XJ, Xia Q, Hou JH, Ding Y, Zhou QM, Zhang X, Pang ZZ, Wan DS, et al: The density of macrophages in the invasive front is inversely correlated to liver metastasis in colon cancer. J Transl Med 8: 13, 2010.

9. Biswas SK and Mantovani A: Macrophage plasticity and interaction with lymphocyte subsets: Cancer as a paradigm. Nat Immunol 11: 889-896, 2010.

10. Mantovani A, Sica A, Sozzani S, Allavena P, Vecchi A and Locati M: The chemokine system in diverse forms of macrophage activation and polarization. Trends Immunol 25: 677-686, 2004

11. Edin S, Wikberg ML, Dahlin AM, Rutegård J, Öberg Å, Oldenborg PA and Palmqvist R: The distribution of macrophages with a M1 or M2 phenotype in relation to prognosis and the molecular characteristics of colorectal cancer. PLoS One 7: e47045, 2012.

12. Chanmee T, Ontong P, Konno K and Itano N: Tumor-associated macrophages as major players in the tumor microenvironment Cancers (Basel) 6: 1670-1690, 2014.

13. Allavena P, Sica A, Solinas G, Porta C and Mantovani A: The inflammatory micro-environment in tumor progression: The role of tumor-associated macrophages. Crit Rev Oncol Hematol 66: $1-9,2008$

14. Yoshikawa F, Banno Y, Otani Y, Yamaguchi Y, NagakuraTakagi Y, Morita N, Sato Y, Saruta C, Nishibe H, Sadakata T, et al: Phospholipase D family member 4 , a transmembrane glycoprotein with no phospholipase D activity, expression in spleen and early postnatal microglia. PLoS One 5: e13932, 2010.

15. Otani Y, Yamaguchi Y, Sato Y, Furuichi T, Ikenaka K, Kitani H and Baba H: PLD4 is involved in phagocytosis of microglia: Expression and localization changes of PLD4 are correlated with activation state of microglia. PLoS One 6: e27544, 2011.

16. Terao C, Ohmura K, Kawaguchi Y, Nishimoto T, Kawasaki A, Takehara K, Furukawa H, Kochi Y, Ota Y, Ikari K, et al: PLD4 as a novel susceptibility gene for systemic sclerosis in a Japanese population. Arthritis Rheum 65: 472-480, 2013.

17. Okada Y, Terao C, Ikari K, Kochi Y, Ohmura K, Suzuki A, Kawaguchi T, Stahl EA, Kurreeman FAS, Nishida N, et al: Metaanalysis identifies nine new loci associated with rheumatoid arthritis in the Japanese population. Nat Genet 44: 511-516, 2012.

18. Krieg T and Takehara K: Skin disease: A cardinal feature of systemic sclerosis. Rheumatology (Oxford) 48 (Suppl 3): iii14-iii18, 2009.
19. Sugihara $H$, Ishimoto $T$, Watanabe $M$, Sawayama $H$, Iwatsuki $M$ Baba Y, Komohara Y, Takeya M and Baba H: Identification of miR-30e* regulation of Bmil expression mediated by tumorassociated macrophages in gastrointestinal cancer. PLoS One 8: e81839, 2013

20. De Palma M and Lewis CE: Macrophage regulation of tumor responses to anticancer therapies. Cancer Cell 23: 277-286, 2013.

21. DeNardo DG, Brennan DJ, Rexhepaj E, Ruffell B, Shiao SL, Madden SF, Gallagher WM, Wadhwani N, Keil SD, Junaid SA, et al: Leukocyte complexity predicts breast cancer survival and functionally regulates response to chemotherapy. Cancer Discov 1: 54-67, 2011.

22. Prada CE, Jousma E, Rizvi TA, Wu J, Dunn RS, Mayes DA, Cancelas JA, Dombi E, Kim MO, West BL, et al: Neurofibromaassociated macrophages play roles in tumor growth and response to pharmacological inhibition. Acta Neuropathol 125: 159-168, 2013.

23. Wang B, Li Q, Qin L, Zhao S, Wang J and Chen X: Transition of tumor-associated macrophages from MHC class II(hi) to MHC class II(low) mediates tumor progression in mice. BMC Immunol 12: 43, 2011.

24. Steidl C, Lee T, Shah SP, Farinha P, Han G, Nayar T, Delaney A, Jones SJ, Iqbal J, Weisenburger DD, et al: Tumor-associated macrophages and survival in classic Hodgkin's Lymphoma. N Engl J Med 362: 875-885, 2010.

25. Hari DM, Leung AM, Lee JH, Sim MS, Vuong B, Chiu CG and Bilchik AJ: AJCC Cancer Staging Manual 7th edition criteria for colon cancer: do the complex modifications improve prognostic assessment? J Am Coll Surg 217: 181-190, 2013.

26. Mantovani A, Biswas SK, Galdiero MR, Sica A and Locati M: Macrophage plasticity and polarization in tissue repair and remodelling. J Pathol 229: 176-185, 2013.

27. Nucera S, Biziato D and De Palma M: The interplay between macrophages and angiogenesis in development, tissue injury and regeneration. Int J Dev Biol 55: 495-503, 2011.

28. Pollard JW: Trophic macrophages in development and disease. Nat Rev Immunol 9: 259-270, 2009.

29. Tang X, Mo C, Wang Y, Wei D and Xiao H: Anti-tumour strategies aiming to target tumour-associated macrophages. Immunology 138: 93-104, 2013.

30. Sica A and Bronte V: Altered macrophage differentiation and immune dysfunction in tumor development. J Clin Invest 117: 1155-1166, 2007.

31. Shime H, Matsumoto M, Oshiumi H, Tanaka S, Nakane A, Iwakura Y, Tahara H, Inoue N and Seya T: Toll-like receptor 3 signaling converts tumor-supporting myeloid cells to tumoricidal effectors. Proc Natl Acad Sci USA 109: 2066-2071, 2012.

32. Pan XQ: The mechanism of the anticancer function of $\mathrm{M} 1$ macrophages and their use in the clinic. Chin J Cancer 31: $557-563,2012$. 